\title{
3D RECONSTRUCTION OF BOTH SHAPE AND BONE MINERAL DENSITY DISTRIBUTION OF THE FEMUR FROM DXA IMAGES
}

\author{
Ludovic Humbert ${ }^{1,2}$, Tristan Whitmarsh ${ }^{1,2}$, Mathieu De Craene ${ }^{2,1}$, Luis Miguel del Río Barquero ${ }^{3}$, \\ Karl Fritscher ${ }^{5}$, Rainer Schubert ${ }^{5}$, Felix Eckstein ${ }^{6}$, Thomas Link ${ }^{7}$, Alejandro F. Frangi ${ }^{1,2,4}$ \\ ${ }^{1}$ Center for Computational Imaging and Simulation Technologies in Biomedicine, Universitat Pompeu Fabra, \\ Barcelona, Spain \\ ${ }^{2}$ Networking Biomedical Research Center - Bioengineering, Biomaterials and Nanomedicine, Barcelona, Spain \\ ${ }^{3}$ CETIR Centre Mèdic, Barcelona, Spain \\ ${ }^{4}$ Institució Catalana de Recerca i Estudis Avançats, Barcelona, Spain \\ ${ }^{5}$ Institute for Biomedical Image Analysis, UMIT, Hall in Tirol, Austria \\ ${ }^{6}$ Institute of Anatomy \& Musculoskeletal Research, PMU, Salzburg, Austria \\ ${ }^{7}$ Musculoskeletal and Quantitative Imaging Research Group, UCSF, California, USA
}

\begin{abstract}
The diagnosis of osteoporosis and the prevention of femur fractures is a major challenge for our society. However, the diagnosis performed in clinical routine from Dual Energy X-ray Absorptiometry (DXA) images is limited. This paper proposes a 3D reconstruction method of both the shape and the Bone Mineral Density (BMD) distribution of the proximal femur from routinely used DXA images. The reconstruction accuracy that can be obtained from single-view and multi-view DXA devices was assessed. This evaluation, from 20 bone specimens and simulated DXA images, highlighted a mean shape accuracy of $1.3 \mathrm{~mm}$ and a BMD accuracy of $4.4 \%$ from a single-view DXA image. A multi-view configuration with 2 views (frontal-sagittal) appeared as a good compromise (mean shape accuracy of $0.9 \mathrm{~mm}$ and BMD accuracy of $3.2 \%$ ). We are currently using this method for in vivo clinical studies in order to improve the diagnosis of osteoporosis and the prevention of femur fractures.
\end{abstract}

Index Terms - 3D reconstruction, Statistical atlas, Dual Energy X-rays Absorptiometry, Bone Mineral Density, Osteoporosis

\section{INTRODUCTION}

Femur fracture due to osteoporosis affects about $18 \%$ of women over 50 years old and accounts for significant morbidity, disability, decreased quality of life, mortality and high economic cost for society [1]. Due to the increasing life expectancy, the World Health Organization (WHO) estimated that the number of osteoporotic femur fractures in the world will increase up to $40 \%$ by 2025 [1]. The diagnosis of osteoporosis and the prevention of the femur fracture is thus a major challenge for our society.

In clinical routine, osteoporosis diagnosis is performed using Dual Energy X-ray Absorptiometry (DXA) [2]. From twodimensional (2D) DXA images, the areal Bone Mineral Density (aBMD, the average BMD measured in the DXA image in a given region) is computed. Following the WHO recommendations [1], the aBMD value is then used in clinical routine to provide a diagnosis of osteoporosis.
However, the 2D measurement of this "projected density" is not accurate enough for a profound analysis of the bone quality. A more sensitive analysis, taking into account both the threedimensional (3D) geometry of the femur and the 3D distribution of the BMD is expected to provide a better assessment of osteoporosis.

Such an analysis can be obtained by Quantitative Computed Tomography (QCT). A calibration phantom used during the CTscan acquisition, allows the conversion of the Hounsfield Units in the CT images, to density values, leading to "quantitative" images of bone density. This 3D analysis leads to an accurate prediction of the mechanical resistance of the femur bone [3] and consequently to a better characterisation of the local bone quality and the fracture risk [4]. However, QCT is associated with important financial costs and high radiation doses for the patient. Consequently, this medical imaging device is not used in clinical routine for osteoporosis follow-up [5].

Therefore methods were proposed to perform a 3D analysis from routinely used DXA images.

3D reconstruction methods from single-view DXA (one frontal view) have not been investigated much. To our knowledge, only Langton et al. [6] proposed a 3D reconstruction method from a single DXA image. This method is however limited to the 3D reconstruction of the shape and, consequently, does not provide a 3D analysis of the BMD distribution.

To overcome these limitations, we recently proposed a 3D reconstruction method based on a statistical atlas incorporating both the femoral shape and the 3D BMD distribution [7]. This atlas was used to acquire a 3D reconstruction from a single DXA image.

On the other hand, DXA commercial systems with a C-arm (such as Hologic Discovery QRD series, Hologic Inc, Bedford, MA, USA) are appearing in the market. Such systems allow the acquisition of several DXA images with various view angles. In this context, research teams proposed 3D reconstruction methods of the femur bone from two orthogonal DXA images [8] or from a set of four DXA images [9]. These methods, however, do not provide a subject-specific 3D reconstruction of the BMD distribution. In addition, the best configuration, in terms of the number of views and the view angles that should be used, is still an issue under de- 
bate. Consequently, the added-value of multiple views in comparison to a single view has not been established yet.

To shed some light into this issue, we present in this paper an extension of our recent developments [7] to the 3D reconstruction of both femur shape and BMD distribution from single-view or multi-view DXA images. Several configurations, from one to four DXA images and with different view angles, were investigated to evaluate the impact on the $3 \mathrm{D}$ reconstruction accuracy.

\section{METHOD}

\subsection{Shape and density statistical model}

As described in our recent research work [7], a statistical model, incorporating both the statistical variations in terms of femoral shape and 3D BMD distribution, was used. This model follows the principles of Active Appearance Models [10] and was automatically constructed from a dataset of QCT scans of femurs. The main steps involved in the construction of this atlas are summarized below.

The model requires a database of QCT acquisitions of proximal femurs. First of all, a reference QCT volume is chosen among all the acquisitions. The femoral shape of this reference volume was segmented using ITK-SNAP [11]. This software provides a semi-automated segmentation algorithm, whose result can eventually be refined by the operator. All volumes are subsequently registered to this reference volume by means of an affine transformation followed by a multi scale B-spline registration whereby the displacement of the control points is constrained to guarantee a diffeomorphic deformation field [12]. For each volume both the affine and non-rigid transformations are applied to the segmented surface mesh of the reference volume, resulting in a surface mesh of the bone for each subject. Generalized Procrustes Analysis aligns the surface meshes and statistical model of the shape is finally obtained by applying Principal Component Analysis (PCA) to the vertices of the surface meshes [13].

Subsequently, to capture only the BMD variations irrespective of shape variations, the previously acquired segmentations are used to deform each volume to the same mean reference shape using Thin Plate Spline interpolation [14]. PCA is then applied to the bone density volumes resulting in a BMD distribution model of femur.

To sum up, the statistical model is thus described by a set of parameters defining the shape and a set of parameters characterizing the BMD distribution (Figure 1).

\subsection{D reconstruction method from DXA images}

Our recent work [7] allows us to acquire a 3D reconstruction (shape and BMD distribution), using the atlas described above, from a single-view DXA image. In this paper, we extend this method to the context of multi-view DXA images. The 3D reconstruction is achieved by searching the parameter space of the statistical models (together with a translation, rotation and uniform scaling) that maximizes the similarity between the DXA images and the Digitally Reconstructed Radiographs (DRRs) generated from the model. Similar to [10], the deformation of the shape model needs to be applied to the BMD distribution model inside the shape. This was achieved by applying a Thin Plate Spline transformation defined by the deformation of the shape. The similarity criterion to be optimized is the average of the mean absolute error obtained between each pair of DXA image and DRR (Figure 2).

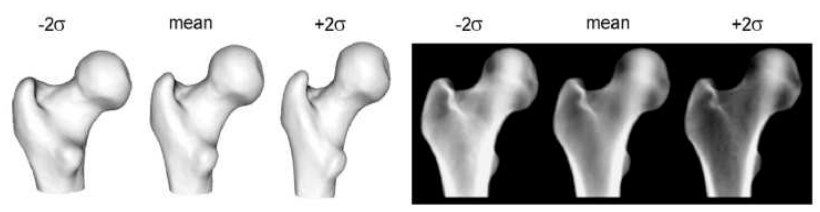

Figure 1: First mode of variation ( \pm 2 standard deviations $\sigma$ from the mean) for the shape (left) and the BMD distribution (right, frontal projection)

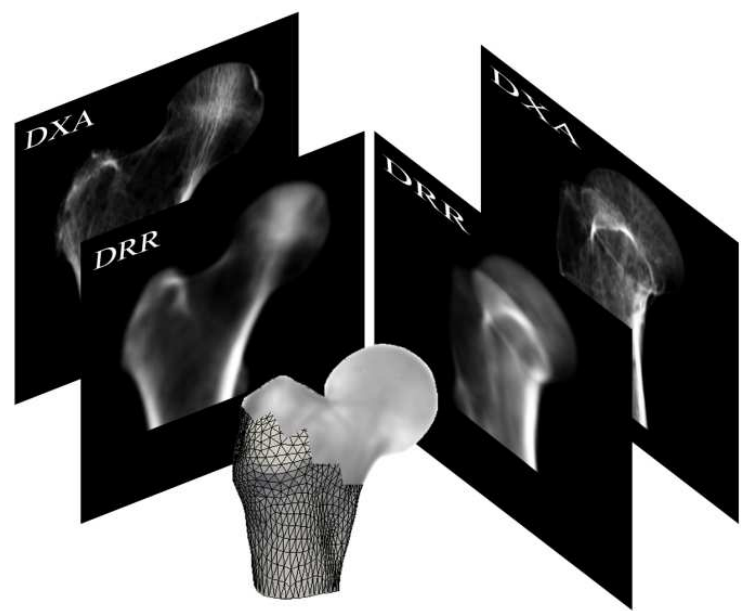

Figure 2: 3D reconstruction from a pair of frontal and sagittal DXA images achieved by maximizing the similarity between the DRRs generated from the statistical model and the DXA images

\section{METHOD EVALUATION}

A database of 64 specimens of human proximal femurs (all female, with a mean age of $80 \pm 10$ years) was collected for a previous study [15] from the institute of Anatomy at the Ludwig Maximilians University Munich (Germany). All these bones were scanned using a 16-row MSCT scanner (Sensation 16; Siemens Medical Solutions, Erlangen, Germany). All the CT-scans were resampled from a spatial resolution of $0.195 * 0.195 * 0.5 \mathrm{~mm}^{3}$ to a spatial resolution of $0.5 * 0.5 * 0.5 \mathrm{~mm}^{3}$ and calibrated using a phantom to obtain a QCT analysis. This database was divided into a first database of 44 samples (mean age: $80 \pm 10$ years) for the construction of the statistical atlas (see section 2.1) and a second database of 20 samples (mean age: $81 \pm 10$ years) for the evaluation of the 3D reconstruction method (see section 2.2). Among these 20 samples, 10 were defined as osteoporotic and 10 as non-osteoporotic based on the WHO criteria [1] (aBMD measurements had been performed from frontal DXA images of the specimens, however, these DXA images have not been stored).

Since the DXA image acquisitions of these specimens were not available, simulated DXA images were generated from the QCT volumes using a ray-casting technique (resolution of $0.3 * 0.3 \mathrm{~mm}^{2}$ ). This technique allowed the generation of realistic DXA images corresponding to true DXA images [16]. This allowed us to easily investigate five different configurations, in terms of the number of simulated DXA images and view angles (enumerated in Table 1).

For each configuration, the $3 \mathrm{D}$ reconstructions obtained from a single or multi-view simulated DXA images were compared with the QCT volumes, which were regarded as the "ground truth". To evaluate the shape accuracy, each of the 20 QCT volumes was 
semi-automatically segmented using ITK-SNAP [11] (as done for the reference shape built in section 2.1). The shapes obtained from the $3 \mathrm{D}$ reconstruction method were subsequently superimposed (Iterative Closest Point method [17]) onto their corresponding segmentation and the point-to-surface distances were computed. In order to estimate the accuracy of the BMD distribution, the reconstructed volume was aligned to the ground truth CT volume using the transformation resulting from the previous Iterative Closest Point registration and the BMD differences were estimated at each voxel.

\section{RESULTS}

The mean shape accuracy, in comparison with the "ground truth" QCT segmentations, was $1.3 \mathrm{~mm}$ from one view and between $0.8 \mathrm{~mm}$ and $0.9 \mathrm{~mm}$ from 2, 3 or 4 views (Table 1). Error maps showing the distribution of the mean shape differences are provided in Figure 3.

The average BMD distribution accuracy, resulting from the voxel by voxel comparison between the reconstructions and the "ground truth" QCT volumes was $81 \mathrm{mg} / \mathrm{cm}^{3}$ from one view and between 53 and $60 \mathrm{mg} / \mathrm{cm}^{3}$ from 2, 3 or 4 views (Table 1). In comparison to the mean range of values in terms of density observed in the QCT volumes $\left(1856 \mathrm{mg} / \mathrm{cm}^{3}\right)$, this mean difference represents errors of $4.4 \%$ (one view) and between 2.9 and $3.2 \%(2,3$ or 4 views).

\section{DISCUSSION}

This study aimed at proposing a 3D reconstruction method of both femur shape and BMD distribution from DXA images. Several studies were performed to recover the femoral shape from DXA images $[6,8,9]$. To our knowledge, the method presented in this paper is the first one that allows a $3 \mathrm{D}$ reconstruction of the BMD distribution, and evaluates the accuracy of the method. In this context, several configurations (single and multi-view DXA images) were investigated.

From only one frontal view, the 3D reconstructions were found quite accurate for both the shape (mean error: $1.3 \mathrm{~mm}$ ) and the BMD distribution (mean error: $4.4 \%$ ) (Table 1). The comparison between the frontal DXA image and the DRR (Figure 4, "1 view") highlighted that the projection of the BMD distribution in the $3 \mathrm{D}$ reconstruction is consistent in comparison to the DXA image.

Regarding the multi-view configurations, the addition of the second view (sagittal) resulted in a gain of accuracy for both the shape (mean error: $0.9 \mathrm{~mm}$ ) and the BMD distribution (mean error: $3.2 \%$ ) (Table 1 and Figure 3). This shape accuracy is similar to the one evaluated in [8] (mean shape accuracy of $0.8 \mathrm{~mm}$ using a 3D reconstruction method from two DXA images; frontal and sagittal). However, this method, limited to the shape reconstruction, required an operator time of 10 minutes, dedicated to manual adjustments of the model [8]. In comparison, our method is fully automated and non-supervised. The addition of other views ( 3 or 4 view configurations) brings a slight gain in terms of accuracy. Figure 4 highlights the consistency between the projections of the BMD distribution and the DXA images (configuration " 3 views. 2 "). Note that the two configuration " 3 views. 1 " and " 3 views. 2 " were equivalent in terms of accuracy.
Table 1: Accuracy of the shape and the BMD distribution

\begin{tabular}{|l|c|c|c|c|}
\hline & \multicolumn{2}{|c|}{$\begin{array}{c}\text { Shape accuracy } \\
(\mathrm{mm})\end{array}$} & \multicolumn{2}{|c|}{$\begin{array}{c}\text { BMD distribution } \\
\text { accuracy }\left(\mathrm{mg} / \mathrm{cm}^{3}\right)^{1}\end{array}$} \\
\cline { 2 - 5 } & $\mathrm{Mean}^{2}$ & $95 \% \mathrm{CI}^{3}$ & Mean $^{2}$ & $95 \% \mathrm{CI}^{3}$ \\
\hline 1 view $0^{\circ}$ & 1.2 & 3.2 & $81(4.4 \%)$ & $278(15 \%)$ \\
\hline 2 views $0,90^{\circ}$ & 0.9 & 2.3 & $60(3.2 \%)$ & $193(10 \%)$ \\
\hline 3 views.1 $0,45,90^{\circ}$ & 0.8 & 2.0 & $55(3.0 \%)$ & $176(9.5 \%)$ \\
\hline 3 views.2 $0,90,-45^{\circ}$ & 0.8 & 2.1 & $56(3.0 \%)$ & $178(9.5 \%)$ \\
\hline 4 views $0,45,90,-45^{\circ}$ & 0.7 & 2.0 & $53(2.9 \%)$ & $171(9.2 \%)$ \\
\hline
\end{tabular}

$1 \%$ related to the range of $B M D$ values observed in the $Q C T$ volumes, ${ }^{2}$ Mean of the absolute differences, ${ }^{3} 95 \%$ Confidence Interval: 2 standard deviation of the signed differences

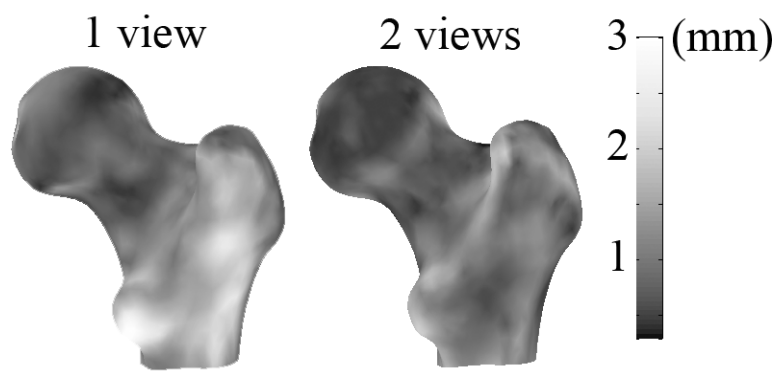

Figure 3: Distribution of the mean shape differences
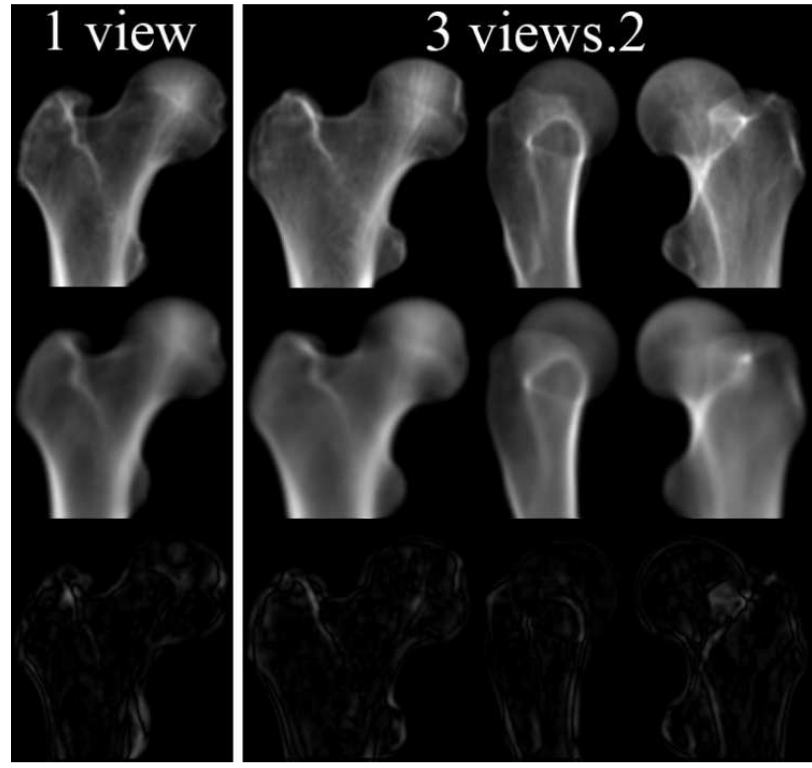

Figure 4: Comparison of the DXA images (top) and the projection of the BMD distribution from the $3 D$ reconstruction (DRRs, middle). Subtraction between the pairs of images (bottom). Representative examples of the mean BMD distribution accuracy (Table 1). 
By relying on the interesting results that we obtained in this in vitro context, this approach is currently evaluated for in vivo clinical applications. Although the evaluation presented in this paper was performed from simulated DXA images, the simulation technique used has been shown to produce realistic simulated DXA images from QCT [16]. Consequently, to apply this method to "true" DXA images is not a major issue. However, with clinical DXA images of patients, we need to deal with the superimposition of other bony structures such as the pelvis. A preliminary evaluation performed from DXA images of patients have recently confirmed that this method provides accurate $3 \mathrm{D}$ reconstructions, even in this in vivo clinical context.

\section{CONCLUSION}

The method proposed in this paper allows the 3D reconstruction of the proximal femur from DXA images with a satisfactory accuracy in terms of shape and BMD distribution. From one DXA image, this method is compatible with the current clinical practice, since most of the clinical sites are equipped with single-view DXA imaging devices. For the multi-view DXA medical systems equipped with a $\mathrm{C}$-arm, that are appearing in clinics, the configuration "2 views" (frontal and sagittal) yield the best compromise. This better characterization of the femoral bone, from clinical routine imaging devices, is expected to provide a better diagnosis of osteoporosis and, consequently, a better prevention of femur fractures. We are currently investigating the potential of this method for such in vivo clinical applications.

\section{ACKNOWLEGDEMENT}

This research has been financially supported by the 3D-FemOs project (3-Dimensional Femur Reconstruction for Osteoporotic Fracture Risk Assessment) with a grant awarded by ACC1Ó Valtec (Tipus 1). In addition this work has been supported by a grant from the Instituto de Salud Carlos III (FI09/00757) and by a grant from the "Deutsche Forschungsgemeinschaft" (LO 730 / 3-1). The authors acknowledge Benedikt Schuler (Institute for Biomedical Image Analysis, UMIT, Hall in Tirol, Austria) and Dr. E. M. Lochmüller (Universitäts-Frauenklinik der LMU, München, Germany) for the data collection.

\section{REFERENCES}

[1] WHO, Prevention and Management of Osteoporosis, World Health Organization Technical Report Series 921, 2003.

[2] A. El Maghraoui, and C. Roux, "DXA scanning in clinical practice," QJM, vol. 101, no. 8, pp. 605-17, Aug, 2008.

[3] K. Fritscher, B. Schuler, T. Link et al., "Prediction of Biomechanical Parameters of the Proximal Femur Using Statistical Appearance Models and Support Vector Regression," Medical Image Computing and Computer-Assisted Intervention - MICCAI 2008, pp. 568-575, 2008.

[4] D. D. Cody, G. W. Divine, K. Nahigian et al., "Bone density distribution and gender dominate femoral neck fracture risk predictors," Skeletal Radiol, vol. 29, no. 3, pp. 151-61, Mar, 2000.

[5] K. Engelke, J. E. Adams, G. Armbrecht et al., "Clinical use of quantitative computed tomography and peripheral quantitative computed tomography in the management of osteoporosis in adults: the 2007 ISCD Official Positions," J Clin Densitom, vol. 11, no. 1, pp. 123-62, Jan-Mar, 2008.

[6] C. M. Langton, S. Pisharody, and J. H. Keyak, "Generation of a 3D proximal femur shape from a single projection 2D radiographic image," Osteoporos Int, vol. 20, no. 3, pp. 455-61, Mar, 2009.

[7] T. Whitmarsh, L. Humbert, M. De Craene et al., "Femur Bone Distribution and Geometry Reconstruction from a Single DXA Image" accepted for SPIE Medical Imaging, 2010.

[8] S. Kolta, A. Le Bras, D. Mitton et al., "Threedimensional X-ray absorptiometry (3D-XA): a method for reconstruction of human bones using a dual X-ray absorptiometry device," Osteoporos Int, vol. 16, no. 8, pp. 969-76, Aug, 2005.

[9] O. M. Ahmad, K. Ramamurthi, K. E. Wilson et al., "3D structural measurements of the proximal femur from 2D DXA images using a statistical atlas," Medical Imaging 2009: ComputerAided Diagnosis. pp. 726005-8, 2009.

[10] T. F. Cootes, G. J. Edwards, and C. J. Taylor, "Active Appearance Models," IEEE PAM. pp. 681-685, 2001.

[11] P. A. Yushkevich, J. Piven, H. C. Hazlett et al., "Userguided 3D active contour segmentation of anatomical structures: significantly improved efficiency and reliability," Neuroimage, vol. 31, no. 3, pp. 1116-28, Jul 1, 2006.

[12] D. Rueckert, P. Aljabar, R. Heckemann et al., "Diffeomorphic Registration Using B-Splines," Medical Image Computing and Computer-Assisted Intervention - MICCAI 2006, pp. 702-709, 2006.

[13] A. F. Frangi, D. Rueckert, J. A. Schnabel et al., "Automatic construction of multiple-object three-dimensional statistical shape models: application to cardiac modeling," IEEE Trans Med Imaging, vol. 21, no. 9, pp. 1151-66, Sep, 2002.

[14] F. L. Bookstein, "Principal Warps: Thin-Plate Splines and the Decomposition of Deformations," IEEE Trans. Pattern Anal. Mach. Intell., vol. 11, no. 6, pp. 567-585, 1989.

[15] F. Eckstein, C. Wunderer, H. Boehm et al., "Reproducibility and side differences of mechanical tests for determining the structural strength of the proximal femur," J Bone Miner Res, vol. 19, no. 3, pp. 379-85, Mar, 2004.

[16] A. Burghardt, G. Kazakia, T. Link et al., "Automated simulation of areal bone mineral density assessment in the distal radius from high-resolution peripheral quantitative computed tomography," Osteoporosis International, in press.

[17] P. Besl, and N. McKay, "A Method for Registration of 3D Shapes," IEEE Trans. Pattern Anal. Mach. Intell., vol. 14, no. 2, pp. 239-256, 1992. 